\title{
Sensitivity to Biases of Case-Control Studies on Medical Procedures, Particularly Surgery and Blood Transfusion, and Risk of Creutzfeldt-Jakob Disease
}

\author{
Jesús de Pedro Cuesta ${ }^{a} \quad$ María Ruiz Tovar $^{\mathrm{a}}$ Hester Ward $^{\mathrm{d}}$ Miguel Calero $^{\mathrm{b}}$ \\ Andrew Smith ${ }^{g}$ Concepción Alonso Verduras ${ }^{c}$ Maurizio Pocchiari ${ }^{\text {h }}$ \\ Marc L. Turner ${ }^{\mathrm{e}}$ Frode Forland ${ }^{\mathrm{i}, j}$ Daniel Palm ${ }^{\mathrm{i}}$ Robert G. Will ${ }^{\mathrm{f}}$ \\ Departments of a Applied Epidemiology, National Centre for Epidemiology and ${ }^{\text {b}}$ Spongiform Encephalopathies, \\ National Microbiology Centre, Consortium for Biomedical Research in Neurodegenerative Diseases (CIBERNED), \\ Carlos III Institute of Health, and 'Blood Products Division, Spanish Medicines and Medical Devices Agency (AEMPS), \\ Madrid, Spain; ' Information Services Division, NHS National Services Scotland, eSchool of Molecular and Clinical \\ Medicine, University of Edinburgh and Scottish National Blood Transfusion Service and ${ }^{\mathrm{f}}$ National CJD Research and \\ Surveillance Unit, Western General Hospital, Edinburgh, and 9 Institute of Infection, Immunity and Inflammation, \\ Glasgow Dental Hospital and School, University of Glasgow, Glasgow, UK; ${ }^{h}$ Department of Cell Biology and \\ Neurosciences, Istituto Superiore di Sanitá, Rome, Italy; ' Office of the Chief Scientist, European Centre for Disease \\ Prevention and Control, Stockholm, Sweden; 'Department of Biomedical Research, Royal Tropical Institute, \\ Amsterdam, The Netherlands
}

Key Words

Creutzfeldt-Jakob disease • Epidemiological studies •

Etiology $\cdot$ Latency analysis

\begin{abstract}
Background: Evidence of risk of Creutzfeldt-Jakob disease (CJD) associated with medical procedures, including surgery and blood transfusion, is limited by susceptibility to bias in epidemiological studies. Methods: Sensitivity to bias was explored using a central-birth-cohort model using data from 18 case-control studies obtained after a review of 494 reports on medical procedures and risk of CJD, systematic for the period January 1, 1989 to December 31, 2011. Results: The validity of the findings in these studies may have been undermined by: recall; control selection; exposure assess-
\end{abstract}

ment in life-time periods of different duration, out of timeat-risk of effect, or asymmetry in case/control data; and confounding by concomitant blood transfusion at the time of surgery. For sporadic CJD (sCJD), a history of surgery or blood transfusion was associated with risk in some, but not all, recent studies at $a \geq 10$ year lag time, when controls were longitudinally sampled. Space-time aggregation of surgical events was not seen. Surgery at early clinical onset might be overrepresented among cases. Neither surgical history nor blood transfusion unlabelled for donor status, dental treatments or endoscopic examinations were linked to variant CJD (vCJD). Conclusions: These results indicate the need for further research. Common challenges within these studies include access to and content of past medical/dental treatment records for diseases with long incubation periods.

Copyright $\odot 2012$ S. Karger AG, Basel

\section{KARGER}

Fax +4161306 1234 E-Mail karger@karger.ch www.karger.com
(C) 2012 S. Karger AG, Base

0251-5350/12/0391-0001\$38.00/0

Accessible online at:

www.karger.com/ned
Jesús de Pedro Cuesta, MD, PhD, Department of Applied Epidemiology

National Centre for Epidemiology and Consortium for

Biomedical Research in Neurodegenerative Diseases

Carlos III Institute of Health, ES-28029 Madrid (Spain)

E-Mail jpedro@isciii.es 


\section{Introduction}

Creutzfeldt-Jakob disease (CJD) is a neurodegenerative disorder with deposition of a pathological isoform (termed $\mathrm{Pr}^{\mathrm{Sc}}$ ) of the normal cellular prion protein $\left(\mathrm{PrP}^{\mathrm{C}}\right)$. CJD exists in three forms: sporadic (sCJD), which is acquired, either variant (vCJD) or iatrogenic (iCJD) CJD, and cases caused by mutations in the gene-encoding $\operatorname{PrP}^{\mathrm{C}}$, here denoted for purposes of simplicity as genetic CJD (gCJD) [1]. The etiology of vCJD has been linked to dietary exposure to bovine spongiform encephalopathy (BSE) or transfusion of blood from infected donors [2, 3]. The cause of SCJD is unknown.

Etiological epidemiological research on vCJD and sCJD related to secondary transmission in the medical settings has been limited. Analytical studies in the UK, encompassing the majority of the reported cases of vCJD, have not indicated an increased risk from surgical or dental procedures $[4,5]$. Studies on medical procedures as a risk factor for SCJD are compromised by methodological limitations $[6,7]$. Results of recent studies on surgical history introducing methodological improvements such as latency analysis, etiological classification of surgical procedures (SP) and SP data obtained from national registries (and, therefore, theoretically independent from recall bias) [8-10], have raised new hypotheses, although space-time relationships between procedures have not been observed [11].

The ultimate goal of this review is to ascertain the scientific basis for specific public health recommendations when drafting EU-wide guidance for the prevention of sCJD transmission in medical settings (ECDC project 1250) [12]. To achieve this, an attempt was made to identify a susceptibility to bias and evidence of risk of CJD associated with medical procedures, including surgery and blood transfusion (BT), in epidemiological studies.

\section{Methods}

In order to identify documents reported from 1989 onwards in English, searches were done using MEDLINE and EMBASE with similar strategies for the period January 1, 1989 to December 31, 2011. First, we adopted a general strategy based on MEDLINE MeSH terms 'prion diseases' OR 'prions' OR 'Creutzfeldt-Jakob syndrome' AND 'case-control studies' OR 'cohort study' or TEXTWORDS 'Creutzfeldt-Jakob', 'case-control' or 'cohort'. Second, findings were added after the EMBASE search, which included EMTREE thesaurus analogous to the above-mentioned MeSH terms, i.e. 'cohort analysis'. Search terms for specific medical procedures or blood-related treatments were not used at this level. After the elimination of dupli- cates, searches provided 506 documents as primary results shown in figure 1. Of these, 34 fulfilled the following two inclusion criteria indicated by quotation marks: mention in the title or abstract of either 'blood transfusion (BT), blood derivatives, blood components, plasma products' or 'diagnostic or therapeutic invasive major and minor medical procedures, endoscopic procedures, ophthalmological procedures, procedures associated with allogeneic tissue implants (dura mater, tendon, etc.)' when applied by at least one of the two reviewers, J.P.C. and M.R.T. The use of exclusion criteria such as letters, reviews, metaanalyses, theoretical issues, methodological papers and documents without abstracts, by a concordant decision by two independent reviewers applying consensus criteria after full document perusal, resulted in the elimination of 6 documents. In order to improve completeness, 5 research reports, 3 of which were published before 1990, and 2 recent papers on BT and surgical history were added [13-17]. Thirty-three documents pertaining to case-control studies and several studied cohorts [410, 13-38] were examined in full text. Thirteen were excluded from the systematic review since: (a) they focused on biographic residential space-time relationships [19]; (b) they were methodological $[6-8,20]$; (c) they focused on the follow-up of national single cohorts of persons exposed to treatment with human pituitary growth hormones in the UK, The Netherlands and the USA [18, 21-24], or with BT or plasma products without data on nonexposed persons $[37,38]$, or $(\mathrm{d})$ they related to human dura mater graft recipients [25]. The search on reports up to 2011 provided 20 full-text documents on 18 case-control studies on CJD where BT or medical procedures were mentioned as potential exposures in the Methods or Results, either as the primary subject of study or as one of a number of potential risk factors, e.g. genetic, occupational or dietary $[4,5,9,10,13-17,26-36]$ (see fig. 1 for details of the selection flow).

The remaining 20 papers related to 18 case-control studies on either sCJD, vCJD or both conditions, and addressed etiological hypotheses including SP and/or BT or history of exposure to plasma products. Some studies generated more than one publication $[9,10,27,28]$. When studies included additional cases or controls the second study was selected for analysis $[15,26]$. We extracted data from Methods and Results, relevant to the study of medical procedures and blood or plasma products, as risk factors in the 18 studies, and we then summarized them.

Sensitivity to biases in the study design for hypotheses related to medical procedures was explored using the study central birth cohort model as proposed by Rothman et al. [39] and Wacholder [40], which was interpreted from a graph, and assessed basic rules of epidemiological analysis, e.g. symmetric exposure assessment, the potential impact of recall or the control of confounding. Medical procedures encompass different interventions such as SP, endoscopies with or without biopsy, dental treatment, transfusion of blood components, or the use of plasma products, all of which involve single exposures. Less frequently there were extended exposures such as coagulation factors in the treatment of hemophilia. In this study the sources of biases that were assessed related mainly to discontinuous exposures and included: (1) type of controls; (2) temporal relationships between case-incidence periods and sampling periods for controls; (3) the source of information on exposures; (4) lifetime references for exposure measurement and individual classification by exposure, and (5) space-time re- 
Fig. 1. Flow diagram of the study selection

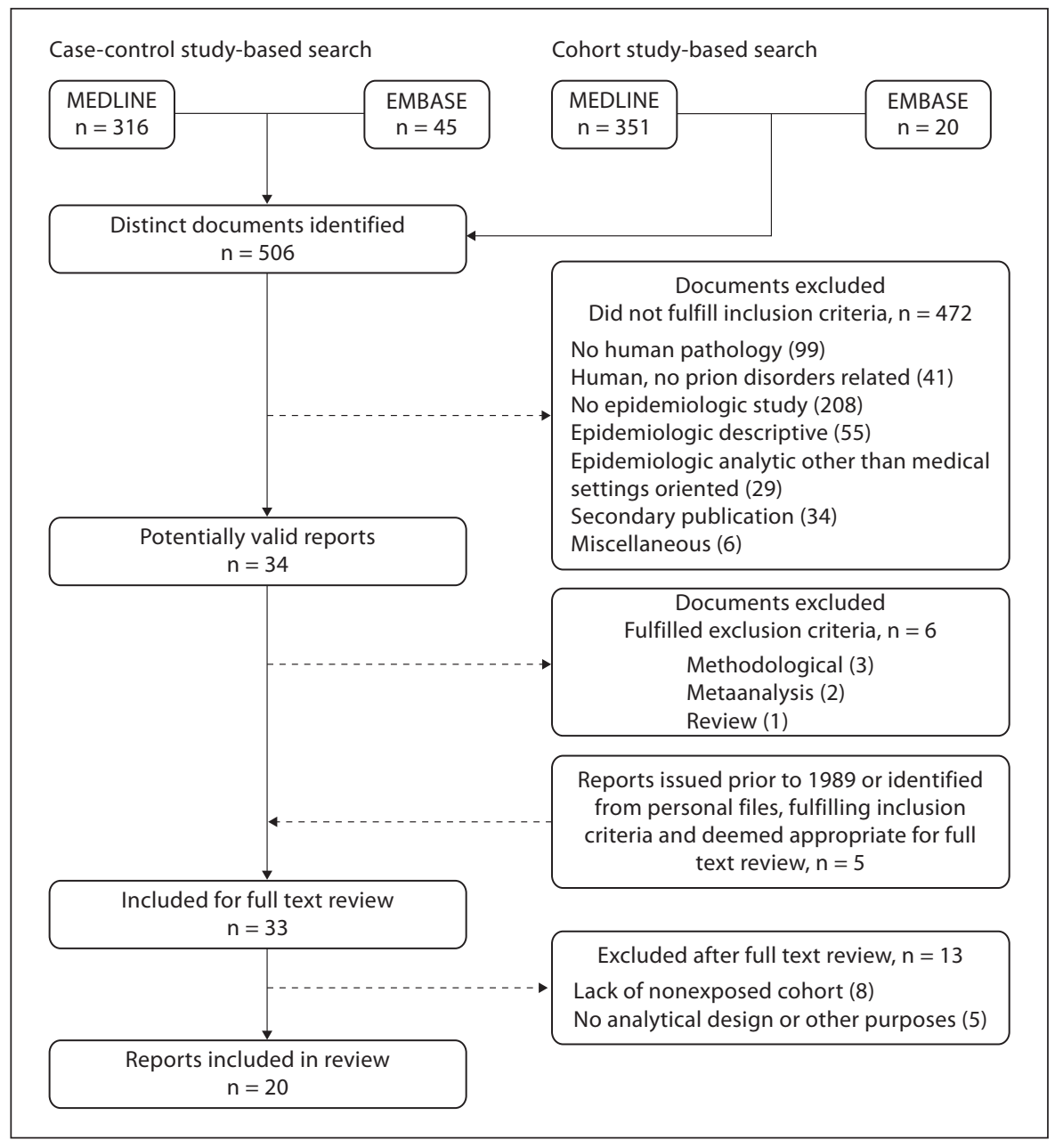
process.

lationship of exposures. The grading of scientific evidence and the application of quality criteria $[41,42]$ in order to support recommendations in public health, based on this paper and other literature reviews, will be reported separately.

\section{Results}

\section{Study Purposes and Domains}

The 20 papers assessed in this report included 1 on CJD (all forms included) [30], 3 on vCJD [4, 5, 33], 17 on sCJD [9, 10, 13-17, 26-29, 31-36] and 1 on gCJD [16]. Seventeen reports focused on surgical history $[4,9,10,13-16$, 27-36] and 14 also included data on BT [4, 13-16, 27-32, 34-36]. Two UK studies addressed BT only [17, 26]. Dental treatment was addressed in 12 reports $[4,5,10,13,14$, $27,28,31,32,34-36]$, and endoscopic procedures in 3 [9, $28,36]$. Other procedures such as tonometry or electro- myography were rarely studied. All the studies were carried out in industrial countries. One was conducted in Australia [31], 3 in Japan [13, 30, 35], 9 in a European country (Germany, Italy, Switzerland and the UK, including vCJD) $[4,5,14,16,17,26,29,34,36]$, and several in two or more European countries [9, 10, 27, 28, 32].

The studies chronologically defined by case recruitment and control sampling are shown in figure 2. Case recruitment in a minority of the studies was before the 1990s but in most it was after 1990, with the study by Esmonde et al. [26] including cases from two separate study periods. In general, earlier studies involved case series and later studies were based on national registries for CJD, which had been established following the identification of BSE in the UK in the late 1980s. Studies based in a single country or in countries with small populations require extended periods for data accrual. Studies from Australia, Italy, Japan and Denmark/Sweden include data 


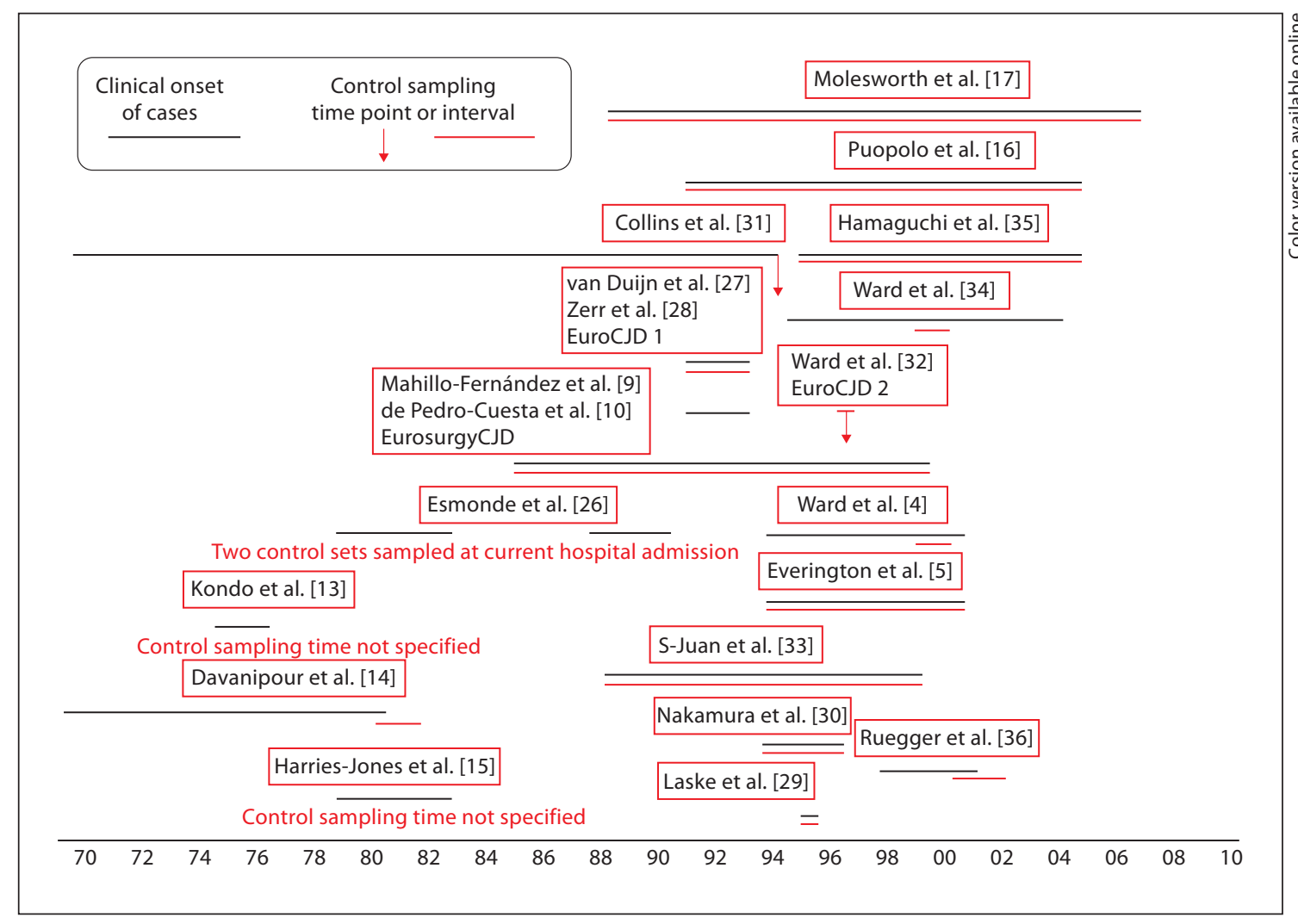

Fig. 2. Outline of study periods defined by the clinical onset of CJD cases and schematic representation, longitudinal or end of study, of the sampling format of controls. Study period symbols underline first-author labels (color online version only).

from extended study periods as defined by the clinical onset of cases $[9,10,16,31,35]$. The studies by countries included in the EuroCJD consortium were carried out over shorter periods, and included different types of controls $[27,28,32]$.

\section{Methodological Aspects}

Methodological details of the 18 case-control studies are summarized in figure 2 and table 1 . In general, multiple hypotheses were examined by the EuroCJD consortium, the Australian and the Swiss studies [27, 28, 31, 32, 36]. Investigators used a questionnaire formulated by the EuroCJD group [5,32], which included questions on diet, contact with animals and occupation, both for sCJD [27, $28,31,32,36]$ and vCJD [4]. A few studies focused on specific hypotheses for sCJD such as BT $[16,17,26]$, surgical events (taken as life stressors) prior to onset [29] and ophthalmological surgery, sometimes including sCJD and vCJD in the UK [33] or dental treatments in the UK for vCJD [5]. Recently, studies by the EurosurgyCJD group were conducted using data on the national populations of Denmark/Sweden. Studies from Italy, Japan, Switzerland and the UK focused on risks related to prior medical history, mainly for medical and SP $[4,9,10,16,34-36]$, some of which included BT $[4,16,17,34-36]$.

In general, controls recruited from hospitals or medical establishments (denoted in this paper as hospital controls) were scarcely used after 1998. Studies from Italy, Japan and the UK used non-CJD cases accrued from CJD surveillance notifications as controls [16, 17, 33, 35]. A UK study, which focused on dental surgery, and a Swiss study used a mix of hospital and population controls [5, 36]. Hospital or primary care-generated controls or a mixed group of hospital and neighborhood controls were included in 11 studies [5, 14-16, 26-30,33,35], and population control sets in 6 studies $[4,9,10,13,31,32,34]$, with 3 sets randomly selected from the study population $[4,9,10,34]$. In general, when details are provided, two patterns emerge (fig. 2). Hospital controls, either from hospitals or reported non-CJD cases, matched or not, were longitudinally 


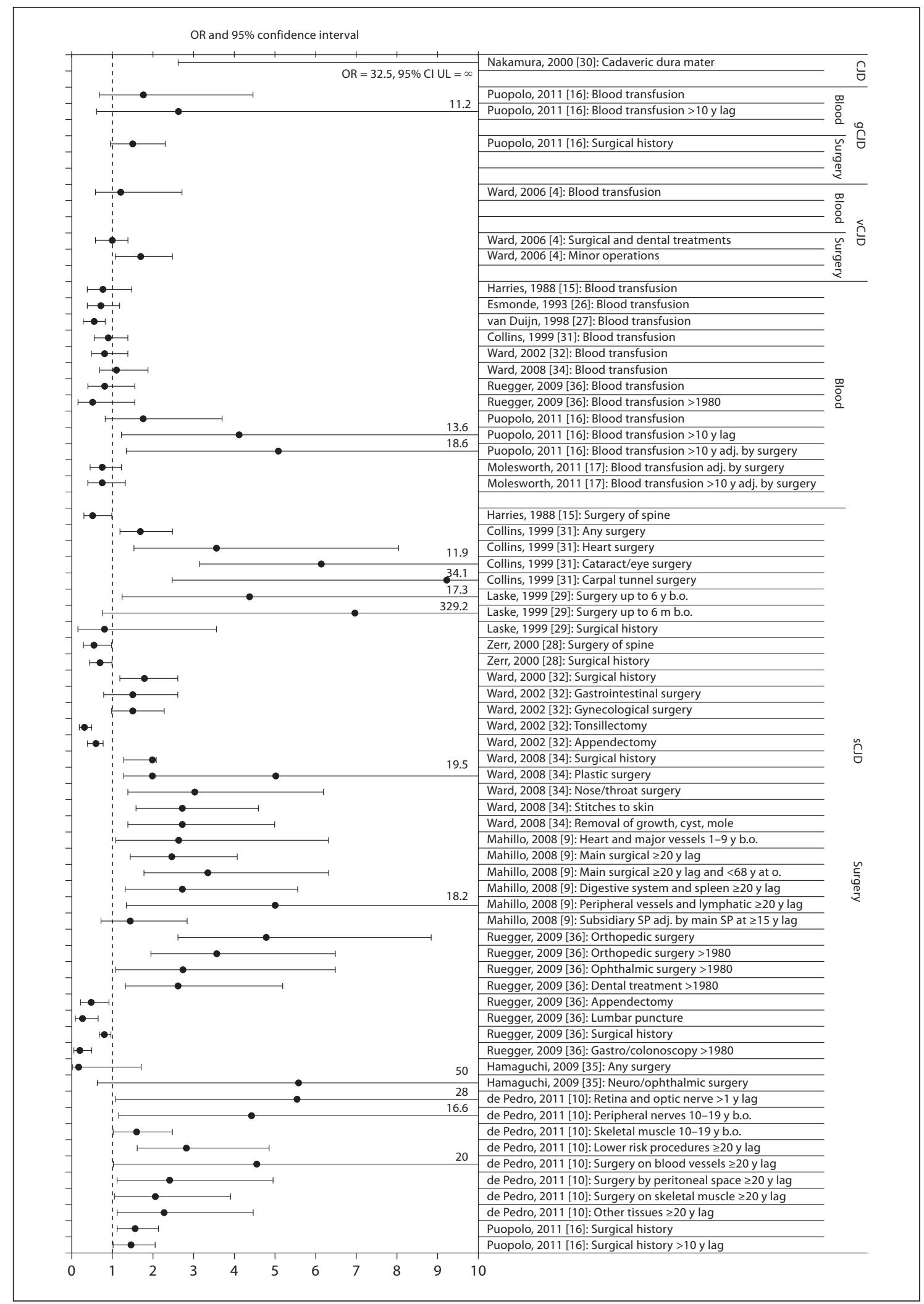

Fig. 3. Graphical representation of selected results. $\mathrm{m}=$ Months; $\mathrm{y}=$ years; $\mathrm{o} .=$ onset; b.o. = before onset; adj. = adjusted. 
Table 1. Methodological features of case-control studies testing associations of medical procedures/surgery/blood with CJD

\begin{tabular}{|c|c|c|c|c|c|}
\hline Authors & $\begin{array}{l}\text { Study purpose } \\
\text { (outcome, tested } \\
\text { hypotheses) }\end{array}$ & $\begin{array}{l}\text { Study base and study } \\
\text { period }\end{array}$ & $\begin{array}{l}\text { Cases } \\
\mathrm{n}\end{array}$ & $\begin{array}{l}\text { Type of } \\
\text { controls }\end{array}$ & $\begin{array}{l}\text { Matching } \\
\text { variables }\end{array}$ \\
\hline $\begin{array}{l}\text { Kondo } \\
\text { et al., } 1982 \\
{[13]}\end{array}$ & $\begin{array}{l}\text { sCJD } \\
\text { Life history } \\
\text { Animal contacts } \\
\text { Medical, surgical and } \\
\text { dental history } \\
\text { BT }\end{array}$ & $\begin{array}{l}\text { Japan } \\
1975-1977\end{array}$ & $\begin{array}{l}60 \text { (37 definite, } \\
23 \text { probable) }\end{array}$ & $\begin{array}{l}56 \text { neighbors } \\
47 \text { spouses }\end{array}$ & Age 5 years, sex \\
\hline $\begin{array}{l}\text { Davanipour } \\
\text { et al., } 1985 \\
{[14]}\end{array}$ & $\begin{array}{l}\text { sCJD } \\
\text { Dietary factors } \\
\text { Medical history } \\
\text { Surgery } \\
\text { Blood } \\
\text { Endodontic procedures }\end{array}$ & $\begin{array}{l}\text { Pennsylvania, USA } \\
\text { and other mid- } \\
\text { Atlantic states }\end{array}$ & $\begin{array}{l}26 \text { (20 definite, } \\
6 \text { probable) }\end{array}$ & $\begin{array}{l}40 \\
18 \text { relatives } \\
22 \text { hospital patients }\end{array}$ & Age 5 years, sex \\
\hline $\begin{array}{l}\text { Harries-Jones } \\
\text { et al., } 1988 \\
{[15]}\end{array}$ & $\begin{array}{l}\text { sCJD } \\
\text { Blood } \\
\text { Medical history } \\
\text { Diet, smoking } \\
\text { Animal contact } \\
\text { Family history of dementia }\end{array}$ & $\begin{array}{l}\text { England and Wales } \\
\text { 1980-1984 }\end{array}$ & $\begin{array}{l}122 \text { (93 definite, } \\
29 \text { probable) }\end{array}$ & $\begin{array}{l}2 \text { per case }(184) \\
\text { Hospital patients } \\
\text { (1 neurological, } 1 \text { medical) }\end{array}$ & $\begin{array}{l}\text { Age } 4 \text { years, sex, } \\
\text { hospital }\end{array}$ \\
\hline $\begin{array}{l}\text { Esmonde } \\
\text { et al., } 1993 \\
{[26]}\end{array}$ & BT & $\begin{array}{l}\text { England and Wales } \\
1980-1984 \text { and UK } \\
\text { CJD surveillance } \\
1990-1992\end{array}$ & $\begin{array}{l}\text { total } 155 \text { cases } \\
\text { ( } 92 \text { definite, } 63 \\
\text { definite or probable) } \\
\text { out of } 202 \text { recruited }\end{array}$ & $\begin{array}{l}184 \text { (see above) } \\
\text { plus } 63 \text { hospital controls } \\
\text { total } 247 \\
\text { hospital controls }\end{array}$ & Age and sex \\
\hline $\begin{array}{l}\text { Van Duijn } \\
\text { et al., } 1998 \text { [27]; } \\
\text { Zerr et al., } \\
2000[28]\end{array}$ & $\begin{array}{l}\text { sCJD } \\
\text { Genetic factors } \\
\text { Lifetime medical history } \\
\text { Blood, dental } \\
\text { Occupational } \\
\text { Animal exposures } \\
\text { Diet }\end{array}$ & $\begin{array}{l}6 \text { European } \\
\text { countries } \\
1993-1995\end{array}$ & $\begin{array}{l}405 \text { (199 definite and } \\
206 \text { probable) }\end{array}$ & 405 hospital patients & $\begin{array}{l}\text { Age } 5 \text { years, sex, } \\
\text { hospital }\end{array}$ \\
\hline $\begin{array}{l}\text { Collins } \\
\text { et al., } 1999 \\
{[31]}\end{array}$ & $\begin{array}{l}\text { sCJD } \\
\text { Medical history } \\
\text { Blood, dental } \\
\text { Others selected: } \\
\text { Occupational, } \\
\text { Residential }\end{array}$ & $\begin{array}{l}\text { Australia } \\
1970-1997 \text { registered }\end{array}$ & $\begin{array}{l}241 \text { (151 definite and } \\
90 \text { probable) }\end{array}$ & $\begin{array}{l}784 \text { population } \\
\text { random telephone dialing }\end{array}$ & $\begin{array}{l}\text { Age } 5 \text { years, sex, } \\
\text { place of } \\
\text { residence }\end{array}$ \\
\hline $\begin{array}{l}\text { Laske } \\
\text { et al., } 1999 \\
\text { [29] }\end{array}$ & $\begin{array}{l}\text { sCJD } \\
\text { Stressful life events: } \\
\text { Psychosocial stress, } \\
\text { Surgery in hospitals, } \\
\text { Medical examinations }\end{array}$ & $\begin{array}{l}\text { Germany } \\
\text { 1997-1998 } \\
\text { surveillance }\end{array}$ & $\begin{array}{l}37 \text { sCJD (7 definite and } \\
30 \text { probable) }\end{array}$ & $\begin{array}{l}37 \text { hospital patients without } \\
\text { dementia (stroke, dizziness, etc.) }\end{array}$ & $\begin{array}{l}\text { Group-matched } \\
\text { by age and sex }\end{array}$ \\
\hline $\begin{array}{l}\text { Nakamura } \\
\text { et al., } 2000 \\
{[25]}\end{array}$ & $\begin{array}{l}\text { CJD } \\
\text { Dura mater grafting } \\
\text { Surgery } \\
\text { BT } \\
\text { Occupation }\end{array}$ & $\begin{array}{l}\text { Japan } \\
\text { 1996-1999 } \\
\text { surveillance }\end{array}$ & $\begin{array}{l}52 \text { out of } 83 \text { CJD } \\
\text { reported cases }\end{array}$ & $\begin{array}{l}102 \text { hospital patients, with 'injuries } \\
\text { and diseases not related to prion' }\end{array}$ & $\begin{array}{l}\text { Age } 2 \text { years, sex, } \\
\text { hospital }\end{array}$ \\
\hline $\begin{array}{l}\text { Ward } \\
\text { et al., } 2002 \\
{[32]}\end{array}$ & $\begin{array}{l}\text { sCJD } \\
\text { Lifetime surgical history } \\
\text { BT }\end{array}$ & $\begin{array}{l}4 \text { European } \\
\text { countries } \\
1993-1995\end{array}$ & $\begin{array}{l}326 \text { (169 definite, } \\
157 \text { probable) }\end{array}$ & $\begin{array}{l}326 \text { population } \\
\text { random telephone dialing in } 2000\end{array}$ & $\begin{array}{l}\text { 5-year age group } \\
\text { and sex } \\
\text { matching, } \\
\text { country }\end{array}$ \\
\hline
\end{tabular}


Table 1 (continued)

\begin{tabular}{|c|c|c|c|c|c|}
\hline \multirow[t]{2}{*}{ Control sampling design } & \multirow[t]{2}{*}{$\begin{array}{l}\text { Source of } \\
\text { information on exposure }\end{array}$} & \multirow{2}{*}{$\begin{array}{l}\text { Strategies for control } \\
\text { for lifetime, person- } \\
\text { year, exposure bias }\end{array}$} & \multirow{2}{*}{$\begin{array}{l}\text { Strategies introduced to } \\
\text { control confounding, } \\
\text { avoiding recall or } \\
\text { improving measurement or } \\
\text { quality of lifetime medical } \\
\text { history }\end{array}$} & \multicolumn{2}{|c|}{$\begin{array}{l}\text { Specific analysis of medical procedures } \\
\text { in cases and controls }\end{array}$} \\
\hline & & & & $\begin{array}{l}\text { latency } \\
\text { analysis? }\end{array}$ & $\begin{array}{l}\text { space-time } \\
\text { analysis of } \\
\text { exposures on } \\
\text { study? }\end{array}$ \\
\hline $\begin{array}{l}\text { Not explicit } \\
\text { Likely absent }\end{array}$ & $\begin{array}{l}\text { Cases = proxy phone } \\
\text { interview } \\
\text { Controls = direct phone } \\
\text { interview }\end{array}$ & Not mentioned & $\begin{array}{l}\text { Controls were } \\
\text { reasonalby healthy } \\
\text { individuals }\end{array}$ & $\begin{array}{l}\text { Operations up to onset } \\
5 \text { and } 10 \text { years before } \\
\text { onset }\end{array}$ & Not reported \\
\hline $\begin{array}{l}\text { Not explicit } \\
\text { Likely absent }\end{array}$ & $\begin{array}{l}\text { Cases = proxy phone } \\
\text { interview } \\
\text { Controls = direct phone } \\
\text { interview }\end{array}$ & Not mentioned & $\begin{array}{l}\text { Mail questionnaire before } \\
\text { interview; same questions } \\
\text { formulated in different } \\
\text { ways }\end{array}$ & $\begin{array}{l}\text { Birth to } 14 \text { years } 15 \\
\text { years to } 3 \text { years before } \\
\text { onset }\end{array}$ & Not reported \\
\hline Longitudinal & $\begin{array}{l}\text { Cases = proxy interview } \\
\text { Controls = proxy } \\
\text { interview }\end{array}$ & $\begin{array}{l}\text { Cases = time to } \\
\text { clinical onset } \\
\text { Controls = time to } \\
\text { current hospital } \\
\text { admission }\end{array}$ & $\begin{array}{l}\text { No defined latency } \\
\text { No strategies } \\
\text { Lifetime; all past medical } \\
\text { events related to current } \\
\text { admission in controls } \\
\text { excluded }\end{array}$ & Not reported & Not reported \\
\hline Longitudinal & $\begin{array}{l}\text { Cases = proxy interview } \\
\text { Controls = proxy } \\
\text { interview } \\
\text { (when possible) }\end{array}$ & Matching & Not mentioned & $\begin{array}{l}\text { Lag-time }>2,>4 \text { and } \\
>10 \text { years }\end{array}$ & Not reported \\
\hline
\end{tabular}

\begin{tabular}{|c|c|c|c|c|c|}
\hline End of study & $\begin{array}{l}\text { Cases = medical record on } \\
\text { site examination } \\
\text { Controls = direct phone } \\
\text { interview by a company }\end{array}$ & $\begin{array}{l}\text { By } 5 \text {-year age } \\
\text { group matching }\end{array}$ & Not mentioned & Not reported & Not reported \\
\hline Longitudinal & $\begin{array}{l}\text { Cases }=\text { interviewers } \\
\text { Controls = interviewers } \\
\text { (both to proxy, close } \\
\text { relatives) }\end{array}$ & $\begin{array}{l}\text { Matching } \\
\text { mean age cases } 66 \\
\text { years, controls } 65 \\
\text { years }\end{array}$ & $\begin{array}{l}\text { Pretest sample examined } \\
\text { using medical records }\end{array}$ & $\begin{array}{l}\text { Last } 6 \text { months } \\
\text { Up to } 8 \text { years before } \\
\text { onset } \\
\text { Lifetime }\end{array}$ & Not reported \\
\hline Longitudinal & $\begin{array}{l}\text { Cases = physicians } \\
\text { Controls = physicians }\end{array}$ & $\begin{array}{l}\text { Matching } \\
\text { Mean age cases } 63.7 \\
\text { years, controls } 62.5 \\
\text { years }\end{array}$ & $\begin{array}{l}\text { Examination of medical } \\
\text { records }\end{array}$ & Not reported & Not reported \\
\hline
\end{tabular}


Table 1 (continued)

\begin{tabular}{|c|c|c|c|c|c|}
\hline Authors & $\begin{array}{l}\text { Study purpose } \\
\text { (outcome, tested } \\
\text { hypotheses) }\end{array}$ & $\begin{array}{l}\text { Study base and study } \\
\text { period }\end{array}$ & $\begin{array}{l}\text { Cases } \\
\mathrm{n}\end{array}$ & $\begin{array}{l}\text { Type of } \\
\text { controls }\end{array}$ & $\begin{array}{l}\text { Matching } \\
\text { variables }\end{array}$ \\
\hline $\begin{array}{l}\text { S-Juan } \\
\text { et al., } 2004 \\
{[33]}\end{array}$ & $\begin{array}{l}\text { sCJD } \\
\text { vCJD } \\
\text { Past ophthalmic } \\
\text { (intraocular, extraocular) } \\
\text { surgery; year, hospital, } \\
\text { type } \\
\text { Space/time links }\end{array}$ & $\begin{array}{l}\text { UK surveillance unit } \\
\text { 1990-2002 }\end{array}$ & $\begin{array}{l}\text { Definite and probable } \\
\text { sCJD } 510 \\
\text { vCJD } 125\end{array}$ & $\begin{array}{l}226 \text { hospital patients } \\
106 \text { population } \\
\text { for sCJD } \\
67 \text { hospital patients } \\
155 \text { population } \\
\text { for vCJD }\end{array}$ & $\begin{array}{l}\text { Age- and sex- } \\
\text { matched }\end{array}$ \\
\hline $\begin{array}{l}\text { Everington } \\
\text { et al., } 2006 \\
{[5]}\end{array}$ & $\begin{array}{l}\text { vCJD } \\
6 \text { categories of dental } \\
\text { treatment from } 1980 \\
\text { onwards }\end{array}$ & UK surveillance unit & $\begin{array}{l}\text { Definite and probable } \\
130 \text { cases out of } 159 \\
\text { reported } \\
\text { up to December } \\
2003\end{array}$ & $\begin{array}{l}\text { Mixed, } 149 \text { community up to } 4 / 1 \\
53 \text { hospital controls for } 66 \text { vs. } 53 \\
\text { cases } \\
\text { not neurological diagnoses }\end{array}$ & $\begin{array}{l}\text { Age ( } \pm 4 \text { years)- } \\
\text { and sex-matched }\end{array}$ \\
\hline $\begin{array}{l}\text { Ward } \\
\text { et al., } 2006 \\
{[4]}\end{array}$ & $\begin{array}{l}\text { vCJD } \\
\text { Dietary, medical, surgical, } \\
\text { dental, BT, invasive, } \\
\text { occupational, contacts } \\
\text { with animals/pesticides }\end{array}$ & UK surveillance unit & $\begin{array}{l}\text { Definite and } \\
\text { probable vCJD } \\
136 \text { reported } \\
1995-2003 \\
\text { Median age at onset } \\
26 \text { years }\end{array}$ & $\begin{array}{l}922 \text { random age-weighted } \\
\text { population sample } \\
>5 \text { years resident in UK with } 1 \\
\text { resident alive relative } \\
\text { median age } 33 \text { years }\end{array}$ & None \\
\hline $\begin{array}{l}\text { Ward } \\
\text { et al., } 2008 \\
{[11]}\end{array}$ & $\begin{array}{l}\text { sCJD } \\
\text { Medical procedures, } \\
\text { mainly surgical } \\
\text { Space/time relations }\end{array}$ & UK surveillance unit & $\begin{array}{l}431 \text { ( } 298 \text { definite, } \\
133 \text { probable) } \\
\text { Median age } 51 \text { years } \\
267 \text { subgroup }\end{array}$ & $\begin{array}{l}454 \text { random sample population } \\
\text { Median age } 67 \text { years }\end{array}$ & None \\
\hline $\begin{array}{l}\text { Hamaguchi } \\
\text { et al., } 2009 \\
{[35]}\end{array}$ & $\begin{array}{l}\text { sCJD } \\
\text { Lifetime medical and } \\
\text { surgical history } \\
\text { Blood }\end{array}$ & Japanese resident & $\begin{array}{l}753 \text { definite or } \\
\text { probable, } 1999-2008 \\
\text { Mean age } 67.7 \text { years }\end{array}$ & $\begin{array}{l}210 \text { reported surveillance controls } \\
\text { Mean age } 59 \text { years }\end{array}$ & None \\
\hline $\begin{array}{l}\text { Ruegger } \\
\text { et al., } 2009 \\
{[36]}\end{array}$ & $\begin{array}{l}\text { sCJD } \\
\text { Lifetime medical history } \\
\text { Drug use } \\
\text { Occupation } \\
\text { Diet }\end{array}$ & $\begin{array}{l}\text { Swiss resident } \\
\text { population at high } \\
\text { sCJD incidence } \\
\text { period }\end{array}$ & $\begin{array}{l}69 \text { cases } \\
61 \text { definite } \\
8 \text { probable } \\
\text { Onset at 2001-2004 }\end{array}$ & $\begin{array}{l}224 \text { controls } \\
69 \text { GP based 2004-2005 } \\
\text { (see exclusion criteria) } \\
155 \text { random telephone dialing, } 2005\end{array}$ & $\begin{array}{l}\text { Age, sex } \\
\text { District and } \\
\text { GP list } \\
\text { Age, sex, residence } \\
\text { by language }\end{array}$ \\
\hline $\begin{array}{l}\text { Mahillo } \\
\text { et al., } 2008 \text { [9]; } \\
\text { De Pedro- } \\
\text { Cuesta et al. } \\
2011[10]\end{array}$ & $\begin{array}{l}\text { sCJD } \\
\text { Surgical and endoscopic } \\
\text { history }\end{array}$ & $\begin{array}{l}\text { Danish and } \\
\text { Swedish } \\
\text { resident populations }\end{array}$ & $\begin{array}{l}167 \\
113 \text { definite } \\
54 \text { probable }\end{array}$ & $\begin{array}{l}\text { Population, } \\
835 \text { random, matched for age, sex, } \\
\text { country } \\
2,224 \text { random }\end{array}$ & $\begin{array}{l}\text { Age, sex, } \\
\text { residence } \\
\text { None }\end{array}$ \\
\hline $\begin{array}{l}\text { Puopolo } \\
\text { et al., } 2011 \\
{[16]}\end{array}$ & $\begin{array}{l}\text { sCJD } \\
\text { gCJD } \\
\text { Lifetime history of BT and } \\
\text { surgery }\end{array}$ & $\begin{array}{l}\text { Italy } \\
\text { surveillance period } \\
1993-2008\end{array}$ & $\begin{array}{l}741 \text { sCJD ( } 563 \text { definite }) \\
\text { Mean age } 67 \text { years } \\
175 \text { gCJD } \\
\text { Mean age } 58 \text { years }\end{array}$ & $\begin{array}{l}482 \text { reported surveillance controls } \\
\text { Mean age } 61 \text { years }\end{array}$ & None \\
\hline $\begin{array}{l}\text { Molesworth } \\
\text { et al., } 2011 \\
{[17]}\end{array}$ & $\begin{array}{l}\text { sCJD } \\
\text { Lifetime history of BT }\end{array}$ & $\begin{array}{l}\text { UK surveillance } \\
\text { period May } 1,1990 \text { to } \\
\text { Dec. } 31,2010\end{array}$ & $\begin{array}{l}1,206 \text { sCJD } \\
\text { (978 studied) } \\
\text { Age not reported }\end{array}$ & $\begin{array}{l}585 \text { reported surveillance controls } \\
\text { (136 studied) } \\
\text { Age not reported }\end{array}$ & None \\
\hline
\end{tabular}


Table 1 (continued)

\begin{tabular}{|c|c|c|c|c|c|}
\hline \multirow[t]{2}{*}{ Control sampling design } & \multirow[t]{2}{*}{$\begin{array}{l}\text { Source of } \\
\text { information on exposure }\end{array}$} & \multirow{2}{*}{$\begin{array}{l}\text { Strategies for control } \\
\text { for lifetime, person- } \\
\text { year, exposure bias }\end{array}$} & \multirow{2}{*}{$\begin{array}{l}\text { Strategies introduced to } \\
\text { control confounding, } \\
\text { avoiding recall or } \\
\text { improving measurement or } \\
\text { quality of lifetime medical } \\
\text { history }\end{array}$} & \multicolumn{2}{|c|}{$\begin{array}{l}\text { Specific analysis of medical procedures } \\
\text { in cases and controls }\end{array}$} \\
\hline & & & & $\begin{array}{l}\text { latency } \\
\text { analysis? }\end{array}$ & $\begin{array}{l}\text { space-time } \\
\text { analysis of } \\
\text { exposures on } \\
\text { study? }\end{array}$ \\
\hline $\begin{array}{l}226(1990-1998) \\
1 / 1 \text { hospital } \\
106(1999-2002) \\
1 / 1 \text { population } \\
67(1996-2002) \\
1 / 1 \text { hospital } \\
155 \text { (since } 1998) \text { up to } \\
4 / 1 \text { community }\end{array}$ & $\begin{array}{l}\text { Relatives, general } \\
\text { practitioners records, } \\
\text { case notes, records, } \\
\text { case files in cases } \\
\text { and controls }\end{array}$ & $\begin{array}{l}\text { In cases } \\
\text { intervals } \\
\text { pre- (number } \\
\text { of years) } \\
\text { and post-clinical } \\
\text { onset }\end{array}$ & $\begin{array}{l}\text { Not } \\
\text { mentioned }\end{array}$ & $\begin{array}{l}\text { Not mentioned } \\
\text { Graph on latency } \\
\text { between last eye } \\
\text { surgery and onset } \\
\text { for cases }\end{array}$ & $\begin{array}{l}\text { Operation } \\
\text { year and } \\
\text { hospital } \\
\text { in cases }\end{array}$ \\
\hline $\begin{array}{l}\text { Sampling: same GP } \\
\text { practices and same } \\
\text { hospital as cases for } \\
\text { community and clinical } \\
\text { controls }\end{array}$ & $\begin{array}{l}\text { Interviews to relatives } \\
\text { using standardized } \\
\text { questionnaires } \\
\text { Categories by review } \\
\text { by dental practitioner }\end{array}$ & $\begin{array}{l}\text { Not } \\
\text { mentioned }\end{array}$ & $\begin{array}{l}\text { Not } \\
\text { mentioned }\end{array}$ & Not reported & Not reported \\
\hline $\begin{array}{l}\text { Post-code sectors, in part } \\
\text { selected clusters matched } \\
\text { to vCJD cases } \\
2002-2003 \\
\text { End of study }\end{array}$ & $\begin{array}{l}\text { Relatives } \\
\text { Cases = nurse surveillance } \\
\text { questionnaire } \\
\text { Controls = National } \\
\text { Centre interviewers, } \\
\text { questionnaire }\end{array}$ & $\begin{array}{l}\text { Proportions } \\
\text { exposed } \\
\text { Controls } \\
\text { adjusted to cases } \\
\text { Birth cohort } \\
\text { distribution }\end{array}$ & $\begin{array}{l}\text { Adjustment by } \\
\text { birth cohort, } \\
\text { sex, residence }\end{array}$ & Not reported & Not reported \\
\hline $\begin{array}{l}\text { Post-code sectors, in part } \\
\text { selected clusters matched } \\
\text { to vCJD cases } \\
2002-2003\end{array}$ & $\begin{array}{l}\text { Cases }=\text { nurse interview to } \\
\text { relatives } \\
\text { Controls = National } \\
\text { Centre interview to } \\
\text { relative }\end{array}$ & $\begin{array}{l}\text { Analysis stratified by } \\
\text { decade of birth }\end{array}$ & $\begin{array}{l}\text { Complementary subgroup } \\
\text { analysis ( } 267 \text { cases onset } \\
2001-2005 \text {, controls } \\
\text { interview } \\
\text { 2002-2003) } \\
\text { Space/temporal analysis }\end{array}$ & $\begin{array}{l}\text { Subgroup analysis of } \\
10 \text {-year periods up to } \\
\geq 20 \text { years before onset } \\
\text { Analysis adjusted for } \\
\text { previous surgery, birth } \\
\text { cohort and region }\end{array}$ & $\begin{array}{l}\text { Surgery in } \\
\text { cases }\end{array}$ \\
\hline $\begin{array}{l}\text { Longitudinal, by } \\
\text { notification time }\end{array}$ & $\begin{array}{l}\text { CJD surveillance and } \\
\text { clinical records }\end{array}$ & $\begin{array}{l}\text { Stratified analysis by } \\
\text { age at onset }\end{array}$ & $\begin{array}{l}\text { Direct perusal of medical } \\
\text { records }\end{array}$ & Not reported & Not reported \\
\hline End of study period & $\begin{array}{l}\text { EUROCJD questionnaire } \\
\text { Proxy interviews, } \\
\text { response rate } 68 \%\end{array}$ & Not mentioned & $\begin{array}{l}\text { GP controls noncompliant } \\
\text { or with severe disease or } \\
\text { were excluded } \\
\text { Exposures before and after } \\
1980\end{array}$ & Not reported & Not reported \\
\hline $\begin{array}{l}\text { Longitudinal, selection } \\
\text { from retrospective } \\
\text { registered resident } \\
\text { population }\end{array}$ & $\begin{array}{l}\text { Operating surgeon or } \\
\text { acting physician at } \\
\text { hospital }\end{array}$ & $\begin{array}{l}\text { Strict control by index } \\
\text { and hospital } \\
\text { discharge dates; } \\
\text { uncontrolled } \\
\text { ambulatory } \\
\text { procedures }\end{array}$ & $\begin{array}{l}\text { Validated residential history } \\
\text { in Sweden } \\
\text { Lack of blood transfusion } \\
\text { data } \\
\text { Use of an etiological } \\
\text { classification of surgical } \\
\text { procedures }\end{array}$ & $\begin{array}{l}>1 \text { year, } 1-9 \text { years, } 10- \\
19 \text { years, } \geq 20 \text { years } \\
\text { (alternatively } 1-14 \\
\text { years and } \geq 15 \text { years) } \\
\text { before onset/index date }\end{array}$ & $\begin{array}{l}\text { Hospital and } \\
\text { date analysis of } \\
\text { surgical } \\
\text { discharges of } \\
\text { cases } \geq 20 \text { years } \\
\text { before onset }\end{array}$ \\
\hline $\begin{array}{l}\text { Longitudinal as reported } \\
\text { to surveillance in } \\
\text { 1993-2008 }\end{array}$ & $\begin{array}{l}\text { Cases = at notification by } \\
\text { phone interview or visit to } \\
\text { treating physician. } \\
\text { Controls = same } \\
\text { procedures and timing }\end{array}$ & $\begin{array}{l}\text { Differences in lifetime } \\
\text { at onset for cases and } \\
\text { controls were assessed }\end{array}$ & $\begin{array}{l}\text { Confounding of blood by } \\
\text { surgical history controlled } \\
\text { at data analysis } \\
\text { Recall effect assessed from } \\
\text { differences in findings for } \\
\text { sCDJ and gCJD }\end{array}$ & $\begin{array}{l}\text { Yes, for exposure at } \\
>10 \text {-year lag }\end{array}$ & $\begin{array}{l}\text { No space-time } \\
\text { analysis }\end{array}$ \\
\hline $\begin{array}{l}\text { Longitudinal as reported } \\
\text { to surveillance during } \\
\text { same period }\end{array}$ & $\begin{array}{l}\text { Cases = data obtained } \\
\text { from surveillance records } \\
\text { Controls = same source }\end{array}$ & $\begin{array}{l}\text { Lifetime at risk of } \\
\text { exposure measured, } \\
\text { not reported }\end{array}$ & $\begin{array}{l}\text { Models adjusted for surgical } \\
\text { history, age at onset, } \\
\text { notification year }\end{array}$ & $\begin{array}{l}\text { Yes, for exposure at } \\
>10 \text {-year lag }\end{array}$ & $\begin{array}{l}\text { No space-time } \\
\text { analysis }\end{array}$ \\
\hline
\end{tabular}


Table 2. Methodological classification of case-control studies by type of controls, consistency and type of measurement assessing exposure status, and (far right column) number (1-4) and type (I-V) of items potentially determining vulnerability to bias

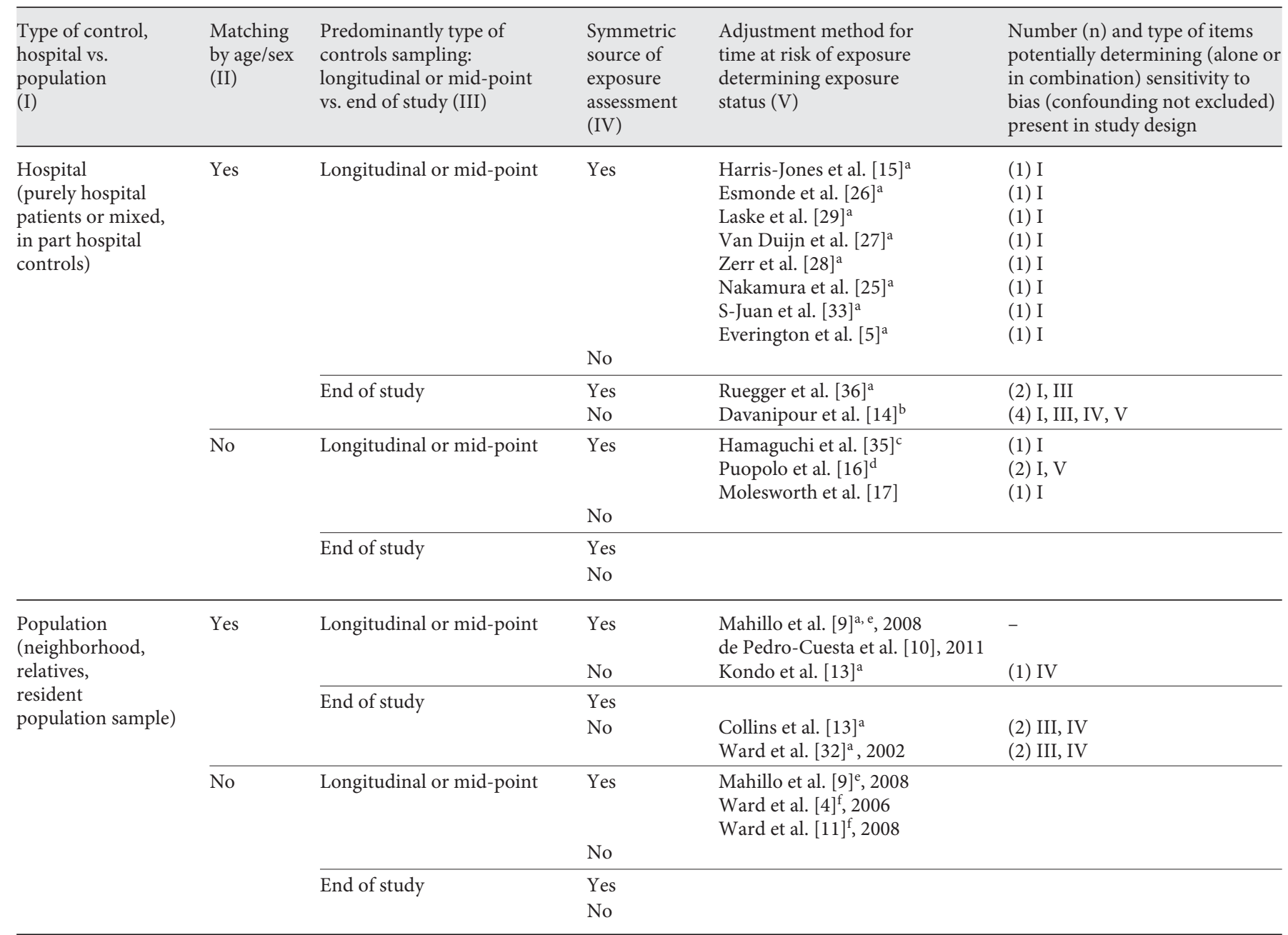

${ }^{\mathrm{a}}$ By matching for age; ${ }^{\mathrm{b}}$ none; ${ }^{\mathrm{c}}$ analysis stratified for age at onset; ${ }^{\mathrm{d}}$ assessed impact of a 6 -year difference in mean age at onset in OR $=5$; ${ }^{\mathrm{e}}$ analysis of equal lifetime intervals by time-windows design; ${ }^{\mathrm{f}}$ by adjusting for birth cohort in the analysis.

sampled [5, 16, 17, 27, 28, 33, 35, 36]. Population controls were sampled at the end $[4,31]$ of the interval for case inclusion [32], except in EurosurgyCJD [9, 10] which, using annual counts of population registries, mimicked longitudinal procedures used in studies with hospital controls.

There was considerable variation in the type of medical procedures assessed. The EuroCJD questionnaire, administered either by direct or telephone interview, was used in approximately half of the studies and produced a standardized list of reported procedures. An inconsistent, i.e. asymmetrical, collection of surgical or BT histories was suggested or evident from descriptions in some studies in which, for example, the controls were the direct informants about their own history [13, 14, 31, 32]. In CJD cases it is rare for the patient to be able to provide any data and this is usually derived from an interview with family members. The time point of data collection was described in only a few studies.

The use of a classification system for diagnoses and procedures was not mentioned except for SP in EurosurgyCJD in which an etiological classification of SP was added to the anatomical one traditionally used in Nordic countries [9, 10]. In reporting an analysis of $\mathrm{BT}$, studies did not mention the differential recording of whole blood, labile components, type of cells or type or number of units. All studies used data on medical history obtained from personal in- 


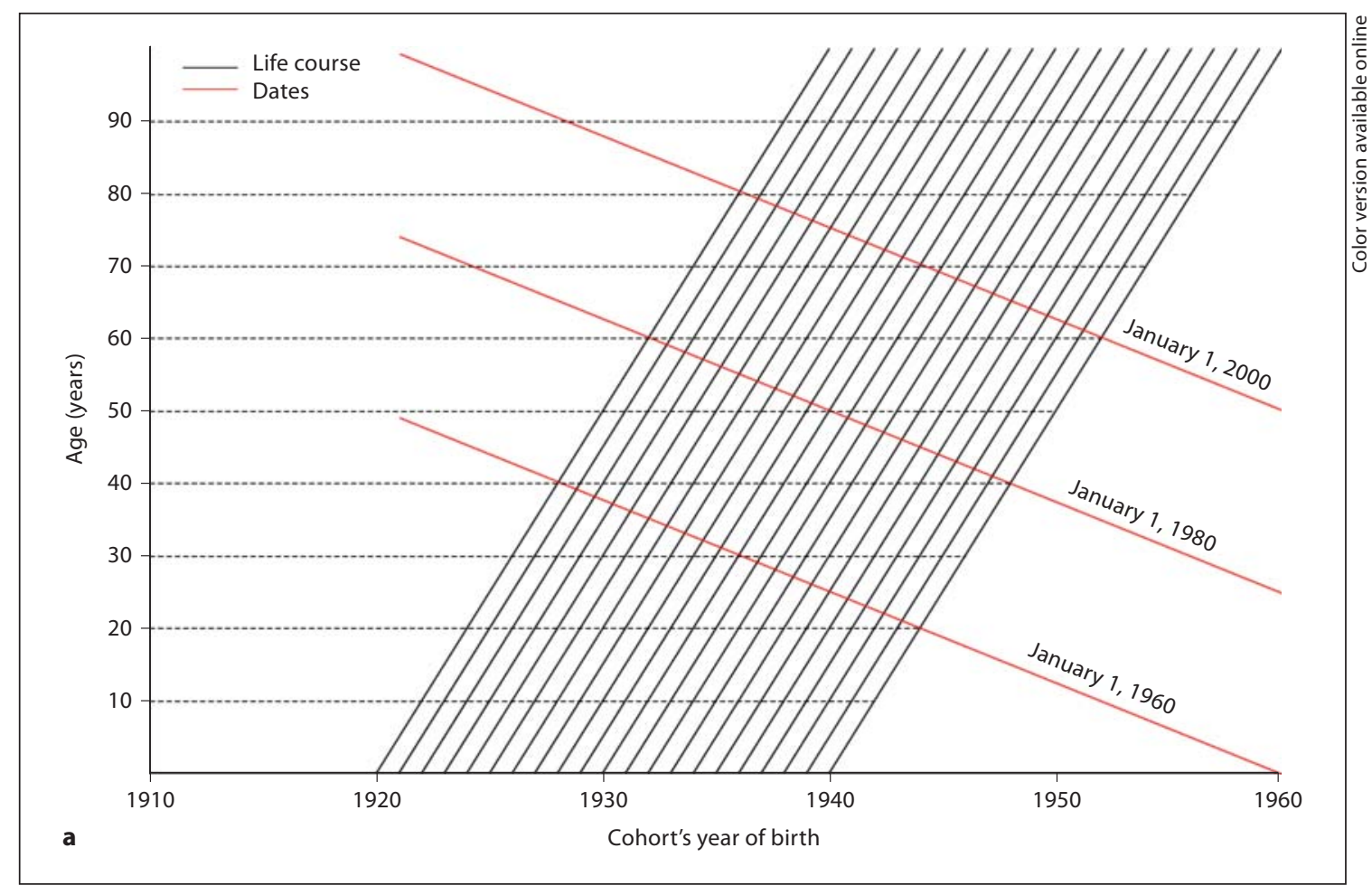

Fig. 4. a Demographic background of a case-control study conducted at end of the 20th century. Ad hoc modified Lexis diagram of the central birth cohort.

terviews, or in some recent studies $[4,16,34,35]$ a retrospective review of hospital and/or general practitioner medical records. Data from national hospital discharge registries with diagnoses and medical procedures coded by specialists at discharge were used in EurosurgyCJD $[9,10]$.

The time reference for exposure assignment in cases, which was often not explicitly stated, encompassed lifelong surgical or BT history up to clinical onset [16, 33, 35], up to 1 year prior to onset $[9,10]$ or up to 3 years prior to onset [28]. Less frequently, authors focused on the period preceding the disease onset and examined surgery during the 2 years prior to onset [28] or during a longer period as in the case of a stressful event in a small German study [29]. A British study of ophthalmic surgery and a Japanese study of surgery in general investigated surgery following disease onset as a putative source of instrument contamination $[33,35]$. The at-risk periods for exposures in the controls were often not explicit, but some studies mentioned lifetime up to interview $[16,32,35]$ or lifetime intervals purposely defined by index dates $[9,10]$.

Consideration of latency or lag periods of different durations from exposure to the onset for risk analysis in sCJD were incorporated in early studies [13, 14], the first
EuroCJD study [28], EurosurgyCJD [9, 10] and recent British and Italian studies $[16,17,34]$. Surgery or medical procedures before or after 1980, a time point related to BSE exposure as a potential etiological factor, were included as a variable in some studies $[4,28,36]$.

Table 2 lists the studies according to the category of exposure assessment in the controls and indicates the types of control groups, the use of age and sex matching, the timing of the interviews in the controls in relation to the cases and whether or not there was symmetric assessment of exposures, i.e. whether information from the controls was obtained directly or indirectly from a family member. Nine reports, the largest group, used hospital, age- and sex-matched controls and consistent exposure assessment. There is a trend in recent studies to include population controls only $[4,9,32,34]$, longitudinal or midpoint sampling $[9,16,17,32,34]$ and consistent exposure assessment $[4,9,10,16,17,34]$.

\section{Findings}

Results for medical procedures, including surgery, BT, endoscopies and dental treatments are described in the online supplementary table (for all online suppl. material, 


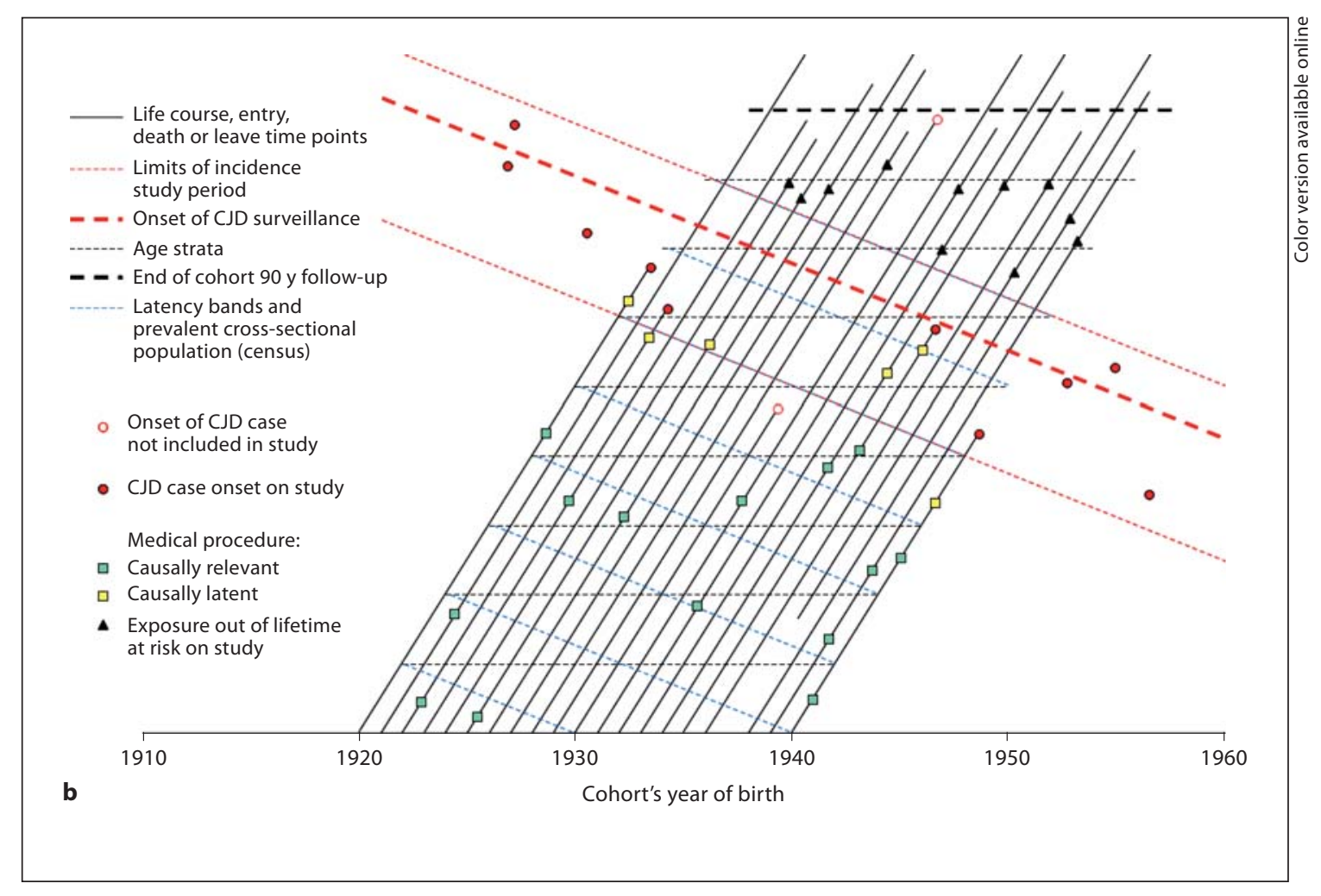

Fig. 4. b Outline of lifetime course and history of medical procedures and CJD diagnoses of a cohort whose members are represented if born during the period 1920-1940. Data for persons pertaining to peripheral birth cohort components are shown only in part (CJD diagnoses, medical procedures). Symbols represent potential time references, CJD diagnoses and time point-related undergone medical procedures. The thin black continuous lines depict the lifetime course, i.e. age in years for persons in the central part of the cohort. The thin black discontinuous bands represent age strata and the parallel thick band at 90 years indicates a hypothetical end of birth cohort follow-up. The graph also describes a follow-up in 2000, at ages ranging from 60 to 80 years for the individuals of the central birth cohort, with events that occurred after this date also indicated. CJD diagnoses are depicted if clinical onsets occurred during the period 1981-2000, i.e. incidence study period. The red discontinuous lines limit the study incidence period and the thin blue discontinuous ones indicate the latency bands, enabling the individually adjusted latency time to be calculated (color online version only). The red discontinuous lines limit time units of the study incidence period and potential controls' sampling intervals. see www.karger.com/doi10.1159/000339318) and figure 3. Data for CJD (all forms included), gCJD and vCJD were sparse $[4,5,16,30]$. Exposure to human dura mater grafts was significantly associated with CJD, odds ratio $(\mathrm{OR})=$ 32 [30]. In 155 cases of gCJD, no risk excess was seen for either BT or surgery at $>10$-year or no lag [16]. In the UK studies of vCJD no association was reported for lifetime surgery except for minor SP and dental extractions, both of which had a wide $95 \% \mathrm{CI}[4,5]$. The proportion of cases and controls with positive BT history, unlabeled for donor status and adjusted by birth cohort (not shown in fig. 3), was similar in both groups [4]. Results for sCJD are summarized as listed below.
Surgery and, when Specified, Invasive Dental

Treatments

With regard to lifetime surgical history, 7 studies with clinical or mixed controls reported no statistically significant associations $[29,33,35]$ or statistically significant protective associations $[15,27,28,36]$. Four studies using community or mixed controls $[31,32,34,36]$ and 1 with hospital controls [16] reported moderately significant associations with lifetime surgical history, OR range 1.62.0. The significant association with no lag persisted at $>10$-year lag [16]. Nonsignificant associations at $>1$-year lag from onset became higher and significant at $\geq 20$ year lag in a register-based study $[9,10]$. In the only study 


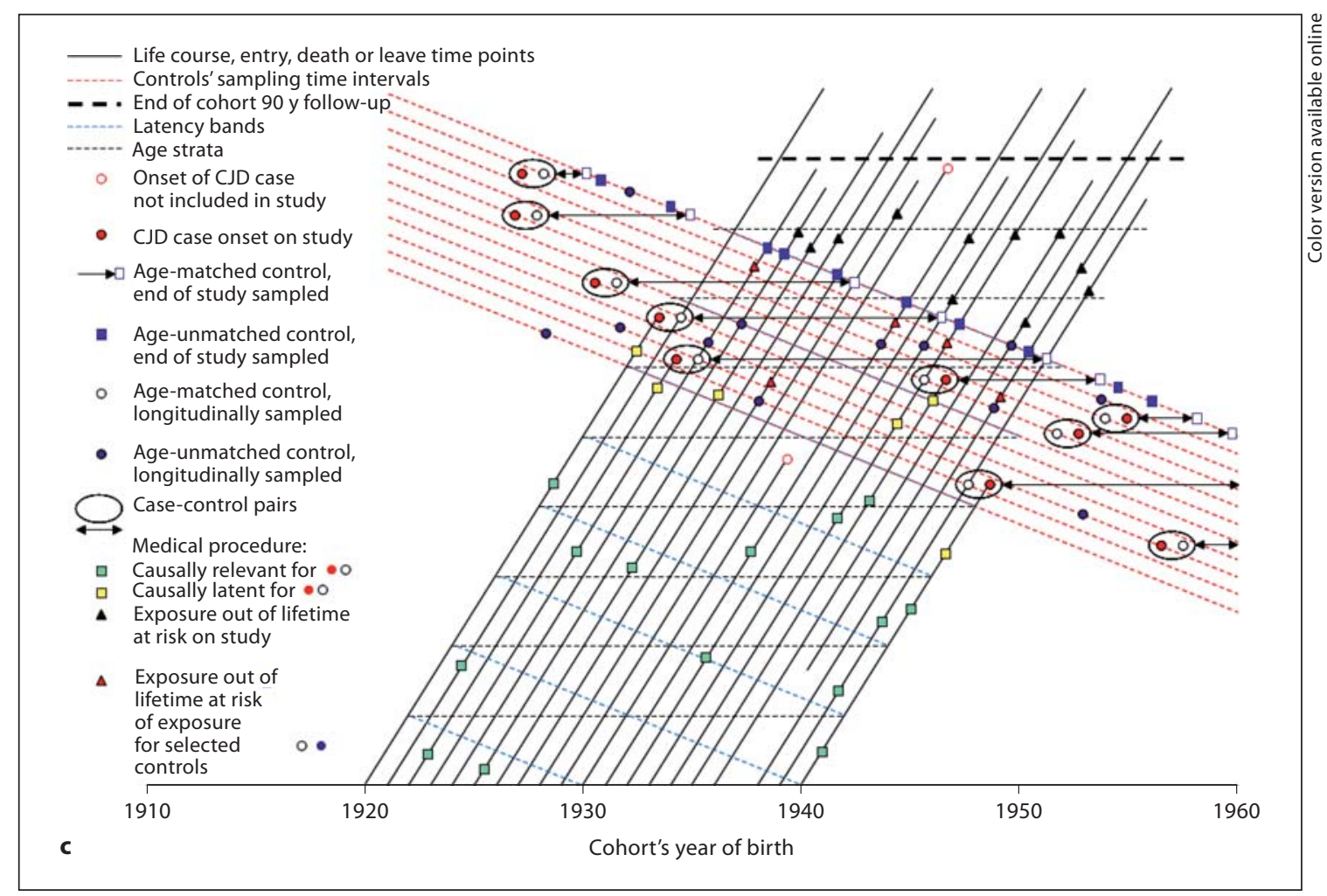

Fig. 4. c Lifetime course and history of medical procedures of a number of individuals (cases, controls, and persons not included in study) selected from the birth cohort corresponding in theory to a case-control study of sCJD with the first cases born in 1920 and the last in 1940 with onsets in the period 1981-2000. Symbols represent potential time references, outcomes and exposures, biologically relevant for specific individuals assuming an incubation period $\geq 20$ years. The thin black continuous lines and the thin blue discontinuous lines represent the time references described in $\mathbf{b}$. The red discontinuous lines limit time units of the study incidence period and potential controls' sampling intervals.

attempting to control confounding, an $\mathrm{OR}=1.45$ at $>10$ year lag for history of surgery became nonsignificant when adjusted for BT at the same time lag [16].

Findings for surgery at specific anatomic sites or settings provided varied results. Few studies had positive findings at no lag: (1) a statistically significant higher frequency of surgery up to 10 years preceding clinical onset was found in EurosurgyCJD, mainly in coronary surgery and after hospital admission in a German study [9, 29]; (2) when using an etiological classification, the only positive association in the EurosurgyCJD study was seen with retinal surgery, which had a mean latency of 9 years to clinical onset $[9,10]$; (3) associations were seen for orthopedic, gastrointestinal and gynecological procedures in studies using community [31,32] or mixed [36] controls, and (4) significant, protective but moderate associations were seen with surgery of the spine in a study using hospital controls $[32,36]$ and with tonsillectomy [32] and appendectomy $[32,36]$ in studies using population and hospital controls, respectively.

At different time lags significant findings varied. A history of surgery of the face, head or neck from the age of 15 years to 3 years before onset was a risk factor for sCJD in an early study in the USA using 'neighbourhood healthy' or a set of clinical and population controls [14]. In EurosurgyCJD, associations were seen for several body system categories of SP with $\geq 20$-year lag and when using an etiological SP classification, at a latency of 10-19 years for surgery of peripheral nerves and in which skeletal muscle was contacted $[9,10]$.

Studies on surgery or medical procedures performed immediately preceding or after clinical onset suggest an excess of ophthalmological [33, 35], neurosurgical [35] and other SP $[29,35]$. 
Only 1 of 7 studies of sCJD and dentistry [13, 14, 27, $28,31,32,36]$ reported a significant positive association with dental treatment, namely for invasive procedures before 1980 [36].

The search for space-time clustering of surgical interventions was investigated for $\mathrm{sCJD}$ cases in some studies with positive results $[9,34]$, but failed to provide evidence of any links. Similar attempts for controls were not mentioned.

\section{Blood and Blood Products}

Findings for a lifetime history of BT in relation to the risk of sCJD were reported in 11 studies. At no lag, protective $(\mathrm{OR}<1)$ effects clearly predominated, being modest but statistically significant in 2, the first EuroCJD and the Swiss studies [27, 28,36], and nonsignificant in 6 studies [13-15, 26, 32, 36]. Other nonsignificant studies include 1 with unreported OR magnitude [35] and 2 British and Italian studies $[16,34]$. An increased risk from BT was found in the only study assessing BT at $\geq 10$-year lag time from onset, which identified statistically significant $\mathrm{OR}=4.09$ and $\mathrm{OR}=5.01$, crude and after adjustment for genotype and surgical history, respectively [16]. These findings were not replicated in another study using a similar methodology [17].

\section{Invasive Diagnostic Procedures}

A protective effect for gastrocolonoscopy was seen in Switzerland, using primary care controls [36]. However, no association was seen either using age-matched clinical controls in the first EuroCJD study $[27,28]$ or after adjusting for surgery in EurosurgyCJD [9].

\section{Discussion}

This review shows that there have been three main risk-based approaches to a potential relationship between medical procedures and CJD, i.e. the case-control design, time-space analysis and cohort studies of persons exposed to recognized iatrogenic factors. The results of these studies are inconsistent and there is no convincing overall evidence that specific medical or SP are associated with an increased risk of developing CJD other than the use of human dura mater grafts. However, all the studies, except perhaps for EurosurgyCJD, have methodological problems that may compromise any definitive conclusions [7], and the body of epidemiological evidence cannot exclude the possibility that surgery or BT may be rarely associated with risk of CJD.
Understanding susceptibility to bias and how this influences the interpretation of results requires a multifaceted approach.

\section{Design}

The study design improved rapidly after vCJD was described and, in many studies since 1998, source populations were identified after national surveillance programs for CJD were officially established. All the studies except EurosurgyCJD $[9,10]$ correspond to the cumulative casecontrol type and address risks such as surgery, which have been defined before control selection begins, i.e. after the potential cases have been identified with the controls selected from noncases [39]. Potentially introduced biases are multiple since (a) a case-control study has been described as 'a cohort study with data missing at random and by design' [40], (b) most studies address lifetime risks and (c) the majority of cases studied in this review were aged 60-79 years at onset during the period 1990-2008 and represent the 1920-1940 birth cohort (figs. 4a, 4b).

Diagnosis and Case Selection. Bias related to case selection, e.g. related to CJD misdiagnosis, is unlikely except in earlier studies in which only a proportion of all cases may have been included $[13,14]$. Overall national mortality rates for SCJD are similar in many countries in which these studies have been carried out and the rates appear to be largely independent of postmortem rates in suspected cases. This suggests that case ascertainment for sCJD has been relatively uniform in many countries in which these studies have been carried out [43]. The impact of potential false-positive diagnoses, such as Alzheimer's disease or Lewy body disease, among cases without pathological confirmation of the diagnosis is unlikely to be an important confounder $[44,45]$.

Control Selection. Some authors have attributed evidence of a protective effect from some medical or SP to be a result of bias related to the use of hospital controls [27] or have introduced compensatory strategies, e.g. excluding surgery related to current hospital admissions in controls [26]. Significant protective effects for lifetime surgery of the spine or other procedures $[15,27,28]$ and for BT $[27,28,30,34]$ found in studies with age- and sexmatched controls, but with negative findings using unmatched hospital controls [35], may be explained by control selection. In contrast, some risk factors [16] may be judged to be truly significant if control groups with minimal potential bias are used.

Matching by age and sex was used in two thirds of the studies $[4,5,9,14,15,26,27,29-34,36]$. Matching constitutes a shortcoming in studies with protracted study pe- 
riods when either population [31] or hospital [14] controls are sampled at the end of the study. The need to identify individuals of the same age at the end of the study requires sampling later birth cohorts who underwent medical procedures at a later calendar time when surgical practices or policy may have changed (see bidirectional arrows in fig. 4c). Bias in such cases is contrary to the policy time trend for the relevant age and sex group. For instance, protective effects from tonsillectomy, a procedure undertaken at a lower mean age in population controls, may be explained by increasing tonsillectomy trends in schoolchildren in the 1930s, as seen at the UK [46]. The death of patients predating the sampling of unmatched control at the end of a 30-year study period may in part underlie some of the associations seen for specific types of surgery in Australia, e.g. cholecystectomy or retina surgery, particularly if there was a decreasing trend for specific types of surgery between 1970 and 2000 [31].

Potential Biases due to Exposure Ascertainment. Medical procedures constitute exposures that may be repeated. In consequence, the individually accumulated exposure to prions can be a complex function of the number and type of medical procedures and the duration of the lifetime interval at risk of exposure. The measurement of exposure frequency is problematic when controls are not matched by age if differences in proportions of persons exposed are due to different average lifetimes at risk of exposure in cases and controls [39]. Even in registerbased studies, such differential bias is likely to be modest and would not explain large ORs, but would lead to an underestimation of risk because of the longer duration of the lifetime at risk of exposure in controls sampled at the end of a study (see exposure events differential between the controls in fig. 4c). Such bias is controlled by creating an index time point close to the longitudinally sampled time units $[9,10]$. The correction is also implicitly done in studies matching for age, and by stratification in unmatched designs, as was done in the CJD surveillancebased Japanese and Italian studies $[16,35]$. Bias inherent in unmatched designs, with population controls identified after the clinical onset in the cases, has been circumvented in two ways: (1) using a genuine longitudinal yearby-year sampling supported by annual national population registries $[9,10]$ or $(2)$ conducting a complementary subanalysis of almost simultaneously identified cases, for 2001-2005, and controls, for 2002-2003, in the UK [34]. Poor record keeping makes studies vulnerable to bias and low statistical power.

Recall is a potential source of bias present in the majority of the reviewed studies for multiple reasons: (1) re- sponses to questions rely on the memory of the informant; (2) the choice or availability of proxy informants varied between studies [13, 14, 31, 32]; (3) since recall accuracy may be influenced by the time elapsed between exposure and recall [47], a further factor may be obtaining information from controls at the end of long study periods [31], and (4) biased responses may be derived from cases as data is obtained after the diagnosis of CJD and respondents may be aware of the hypotheses being tested.

Control of Confounding. SP are frequently associated with other procedures, e.g. transluminal endoscopies, biopsies or BT, and these potential confounders have been controlled for in only 2 studies $[9,16]$. The findings from EurosurgyCJD suggest that the risk from endoscopies is low [9]. In a recent Italian study all the evidence of risk due to surgery was removed by controlling for the effect of BT, suggesting that BT explained the apparent risk excess of concomitant surgery [16]. Public health recommendations for blood or plasma products and invasive procedures should consider both confounded and independent risk effects.

Biological Covariates Determining Effect Measures: Latency and Age. A protracted latency in CJD can be assumed from clinical observations on iCJD and experimental studies [21, 23, 24, 48]. Latency analysis is essential since lifetime frequency of surgery was high in cases (89\%) and in controls (79\%) [34] and unexposed individuals are rare. Latency analysis has provided positive results for surgery and BT at $>10$-year lag in 2 studies [9, 16], 1 of which was register-based [9], but not in the 2 UK studies $[4,17]$, and at $<10$-year lag for surgery in 2 studies $[9,29]$. Currently two thirds of surgical interventions in Swedish hospitals, and probably in other Western countries, occur over the age of 50 years [9]. Most of these procedures would not be relevant to CJD causation at $>20$-year latency, particularly if the risk decreases with a more advanced age at surgery as suggested by EurosurgyCJD, and due to $<20$-year survival [9]. The fact that $30-50 \%$ of SP or BT exposures occur at a more advanced age and are not likely to be relevant to the cause of CJD will have an impact on risk assessment when neglecting latency, i.e. diluting the effect from procedures undergone earlier in life, which may have a higher inherent risk. The majority of studies do not include a latency analysis at $>10$-year lag, and many use hospital controls or controls sampled at the end of the study period. These factors, together with analysis of SP carried out more frequently with time, may lead to an underestimation of risk for SP and BT. 
Age appears to be a biological risk modifier for human prion diseases since the incidence of sCJD or vCJD varies with age. The effect of age at onset is taken into account in many studies by matching or in analysis. However, age may have a complex effect in case-control studies. For example, the risk of sCJD increases with a lower age at surgery [9], and the risk of iCJD associated with human pituitary growth hormone treatment is highest in the 8 to 10 -year age group [21], suggesting that susceptibility to CJD is influenced by age at exposure. An age-related susceptibility function was found to be necessary to reproduce the characteristics of the age distribution of vCJD cases in the UK [49]. Furthermore, age at exposure to surgery and age at onset of sCJD were found to be directly associated [9]. In consequence, three age-related effects need to be independently considered in CJD studies: (1) age at exposure to medical procedures, (2) age at clinical onset and (3) age at the time of exposure assessment, which determines the length of the studied lifetime interval. All three factors should be evaluated as possible sources of bias in the quality assessment of studies which are used for supporting recommendations for risk prevention.

\section{Conclusion}

This review reveals a number of potential methodological pitfalls in case-control studies on CJD. Susceptibility to bias may be due to (a) the use of hospital controls and sampling controls close to or at the end of long case incidence periods, (b) exposure assessment in different lifetime periods for cases and controls or disregarding time-at-risk effects and (c) potential confounding by concurrent procedures, particularly surgery and intra-operative BT. One way to synthesize the findings in these studies, which are summarized in figure 3 , is to focus on specific procedures or analyze groups of associations. The interpretation of the biological significance of statistical association patterns requires consideration of experimental and social research, e.g. evidence from laboratory transmission studies and the temporal evolution of surgical technology. These issues are mentioned in some studies $[9,10,16]$ and are outside of the scope of this review.

In the context of the caveats described above, which would preclude conducting any metaanalysis prior to a quality assessment of specific studies, the conclusions below may be suggested after a review of the case-control studies in CJD.
Despite compelling evidence of case-to-case transmission of vCJD by BT $[3,50]$, case-control studies do not disclose an excess risk of vCJD from previous BT (or surgery and dentistry) $[4,5]$. This underlines the limitations in the case-control methodology in identifying rare causal exposures and indicates that negative findings in casecontrol studies in other forms of CJD cannot exclude the possibility of infrequent transmission events.

Case-control data on medical procedures in $\mathrm{CCJD}$ and gCJD are scarce $[16,30]$. One study quantifying the association of human dura mater grafts with CJD confirms a causal relationship, which is supported by clear clinical evidence [30].

In sCJD there are several positive associations with decreasing biological plausibility:

(1) Although there is unconfirmed evidence of stress as a clinical trigger for sCJD [29], an increased frequency of surgery during the prodromal phase or early clinical course in SCJD is likely to be a consequence of the clinical features of the disease. These were quantified in 1 small study [29] and suggested in 2 other studies in which evidence of the clinical indications for surgery were provided $[33,35]$, e.g. CNS neoplasms or cataracts presenting with clinical features that mimicked sCJD. These findings are consistent with experience from clinical observations in SCJD and sCJD surveillance programs.

(2) Positive associations for SP and BT, with the strongest evidence of an association based on latency analysis at $>10$ - or $>20$-year lag $[9,10,16,34]$, are consistent with the protracted incubation periods in human prion disease, but may involve organs or tissues that are not known to be infectious in SCJD $[9,10]$. Although casecontrol studies must be interpreted with caution because of the potential for systematic sources of error [16], the positive findings in relation to SP and BT may indicate a true risk for $\mathrm{SCJD}$, although there may be confounding of SP by BT as mentioned above. Positive associations obtained from studies with less rigorous methodologies [31] should be interpreted with caution.

(3) Positive associations with specific procedures at a shorter latency have been reported recently, e.g. retinal surgery with about a mean 10 -year lag or coronary surgery in the decade preceding $\mathrm{sCJD}$ onset $[9,10]$. The interpretation of these findings requires an assessment of specific, speculative hypotheses. Possibilities include the direct spread of prions to the retina, or the confounding by risk factors shared by coronary disease and sCJD $[9,10]$, as an issue of debate in coronary bypass surgery and Alzheimer's disease $[51,52]$. 
(4) Inverse associations, particularly if obtained from studies with the above-mentioned potential sources of systematic error, are most likely attributable to bias $[27,28]$.

(5) The negative findings for neurosurgery may be explained by limited or inappropriate reference groups (exposed to other surgery) or by the use of hospital controls $[28,32]$, or perhaps because the risk of transmission through potentially contaminated neurosurgical instruments has been reduced by modern decontamination techniques.

There is limited evidence from case-control studies of an excess risk for the development of sCJD from BT and from general surgery after long latencies, and of an excess of surgery close to clinical onset. There is no epidemiological evidence of a risk from invasive dental treatments and endoscopic procedures. This evidence has to be judged in relation to other evidence, e.g. negative lookback studies of BT in SCJD [37]. This review suggests that there is a need for further research and an evidence-based review of policies for the prevention of sCJD in medical settings, for example in EU countries.

\section{Acknowledgements}

The authors are grateful to Javier Almazán, Fuencisla Avellanal and in particular Enrique Alcalde at the CJD Spanish Registry and Department of Applied Epidemiology at CNE, Carlos III Institute of Health, for their efficient and continuous technical support with literature searches, updates, document management and graph layout. We wish to thank Isabel Iribarren, Carlos III University for her early support in search of documents and Ana Belen Escriva, medical librarian at ECDC, for the completion and supervision of searches in MEDLINE and EMBASE.

We are grateful to María Ángeles Pérez, Almudena Flores, Almudena Olivares and Ana María Cochón from Fundación CIEN and CIBERNED for their administrative support.

We would also like to thank members of the consultant group for their comments and criticisms: Elias Sanz, Carlos III University, Madrid; Outi Lyytikainen, Finnish Institute of Public Health, Helsinki; Alberto Rábano, Fundacion CIEN, Madrid; Rosario Arrieta Gallástegui, University Hospital La Paz, Madrid; Odorina Tello Anchuela, National Centre for Epidemiology, ISCIII, Madrid; Pablo Martínez Martín, Carlos III Institute, Madrid; Pascual Sánchez-Juan, Hospital Universitario Marqués de Valdecilla, Santander; Åke Siden, Huddinge University Hospital, Stockholm; Nicky Connor, UK CJD Incidents Panel, Health Protection Agency, London; Sophie Quolin, CJD Registry, Brussels; Hans Blystad, National Institute of Infectious diseases, Oslo; Juan Martínez Lage, Hospital Virgen Arriaxaca, Murcia; Mabel Cruz, Karolinska Institute, Stockholm; Paul Brown, USA, and Margarita Ramírez de Santa Pau, Comunidad Autónoma Madrid.

This study was partially supported by CIBERNED and the European Centre for Disease Prevention and Control, Specific Contract ECDC 1250. The ECDC provided funding for the EuroCJD consortium.

\section{Disclosure Statement}

The authors declare no conflicts of interest. Prof. Andrew Smith received funding for a $\mathrm{PhD}$ studentship from a company called W\&H Ltd. (manufacturers of dental hand pieces and bench top steam sterilizers). In the past he has received lecture fees and travel expenses from Steris Ltd. and Schulke \& Mayr.

\section{References}

Prusiner SB: The prion diseases. Brain Pathol 1998;8:499-513.

-2 Bruce ME, Will RG, Ironside JW, McConnell I, Drummond D, Suttie A, et al: Transmissions to mice indicate that 'new variant' CJD is caused by the BSE agent. Nature 1997;389: 498-501.

3 Hewitt PE, Llewelyn CA, Mackenzie J, Will RG: Creutzfeldt-Jakob disease and blood transfusion: results of the UK Transfusion Medicine Epidemiological Review study. Vox Sang 2006;91:221-230.

-4 Ward HJ, Everington D, Cousens SN, SmithBathgate B, Leitch M, Cooper S, et al: Risk factors for variant Creutzfeldt-Jakob disease: a case-control study. Ann Neurol 2006;59: $111-120$.

5 Everington D, Smith AJ, Ward HJ, Letters S, Will RG, Bagg J: Dental treatment and risk of variant CJD - a case control study. Br Dent J 2007;202:E19, discussion 470-471.
-6 Riggs JE, Moudgil SS, Hobbs GR: CreutzfeldtJakob disease and blood transfusions: a meta-analysis of case-control studies. Mil Med 2001;166:1057-1058.

7 Barash JA, Johnson BT, Gregorio DI: Is surgery a risk factor for Creutzfeldt-Jakob disease? Outcome variation by control choice and exposure assessments. Infect Control Hosp Epidemiol 2008;29:212-218.

8 de Pedro-Cuesta J, Bleda J, Rabano A, Cruz $\mathrm{M}$, Laursen H, Molbak K, et al: Classification of surgical procedures for epidemiologic assessment of sporadic Creutzfeldt-Jakob disease transmission by surgery. Eur J Epidemiol 2006;21:595-604.

-9 Mahillo-Fernandez I, de Pedro-Cuesta J, Bleda J, Cruz M, Molbak K, Laursen H, et al: Surgery and risk of sporadic Creutzfeldt-Jakob disease in Denmark and Sweden: registry-based case-control studies. Neuroepidemiology 2008;31:229-240.
0 de Pedro-Cuesta J, Mahillo-Fernandez I, Rabano A, Calero M, Cruz M, Siden A, et al: Nosocomial transmission of sporadic Creutzfeldt-Jakob disease: results from a risk-based assessment of surgical interventions. J Neurol Neurosurg Psychiatry 2011; 82:204-212.

11 Ward HJ, Knight RS: Surgery and risk of sporadic Creutzfeldt-Jakob disease. Neuroepidemiology 2008;31:241-242.

12 Ruiz-Tovar M, de Pedro-Cuesta J, Smith A, Alonso C, Calero M, Pocchiari M, et al: Review of guidelines for prevention of Creutzfeldt-Jakob disease transmission in medical settings in EU member states and Norway. Stockholm, ECDC Technical Report, 2011. http://www.ecdc.europa.eu/en/ publications/Publications/1106_TER_Review_of_guidelines_for_prevention_of $\% 20$ CJD.pdf (cited 2011 June 21). 
13 Kondo K, Kuroiwa Y: A case control study of Creutzfeldt-Jakob disease: association with physical injuries. Ann Neurol 1982;11:377381.

-14 Davanipour Z, Alter M, Sobel E, Asher D, Gajdusek DC: Creutzfeldt-Jakob disease: possible medical risk factors. Neurology 1985;35:1483-1486.

-15 Harries-Jones R, Knight RS, Will RG, Cousens SN, Smith PG, Matthews WB: Creutzfeldt-Jakob disease in England and Wales, 1980-1984: a case-control study of potential risk factors. J Neurol Neurosurg Psychiatry 1988;51:1113-1119.

-16 Puopolo M, Ladogana A, Vetrugno V, Pocchiari M: Transmission of sporadic Creutzfeldt-Jakob disease by blood transfusion: risk factor or possible biases. Transfusion 2011;51:1556-1566.

-17 Molesworth AM, Mackenzie J, Everington D, Knight RS, Will RG: Sporadic CreutzfeldtJakob disease and risk of blood transfusion in the United Kingdom. Transfusion 2011;51: 1872-1873.

-18 Abrams JY, Schonberger LB, Belay ED, Maddox RA, Leschek EW, Mills JL, et al: Lower risk of Creutzfeldt-Jakob disease in pituitary growth hormone recipients initiating treatment after 1977. J Clin Endocrinol Metab 2011;96:E1666-E1669.

19 Linsell L, Cousens SN, Smith PG, Knight RS, Zeidler M, Stewart G, et al: A case-control study of sporadic Creutzfeldt-Jakob disease in the United Kingdom: analysis of clustering. Neurology 2004;63:2077-2083.

-20 Rabano A, de Pedro-Cuesta J, Molbak K, Siden A, Calero M, Laursen H: Tissue classification for the epidemiological assessment of surgical transmission of sporadic Creutzfeldt-Jakob disease. A proposal on hypothetical risk levels. BMC Public Health 2005;5:9.

-21 Swerdlow AJ, Higgins CD, Adlard P, Jones ME, Preece MA: Creutzfeldt-Jakob disease in United Kingdom patients treated with human pituitary growth hormone. Neurology 2003;61:783-791.

-22 Wientjens DP, Rikken B, Wit JM, Hofman A, Stricker BH: A nationwide cohort study on Creutzfeldt-Jakob disease among human growth hormone recipients. Neuroepidemiology 2000;19:201-205.

-23 Buchanan CR, Preece MA, Milner RD: Mortality, neoplasia, and Creutzfeldt-Jakob disease in patients treated with human pituitary growth hormone in the United Kingdom. BMJ 1991;302:824-828.

24 Mills JL, Fradkin JE, Schonberger LB, Gunn W, Thomson RA, Piper J, et al: Status report on the US human growth hormone recipient follow-up study. Horm Res 1990;33:116-119.

-25 Nakamura Y, Aso E, Yanagawa H: Relative risk of Creutzfeldt-Jakob disease with cadaveric dura transplantation in Japan. Neurology 1999;53:218-220.
26 Esmonde TF, Will RG, Slattery JM, Knight RS, Harries-Jones $\mathrm{R}$, de Silva $\mathrm{R}$, et al: Creutzfeldt-Jakob disease and blood transfusion. Lancet 1993;341:205-207.

27 van Duijn CM, Delasnerie-Laupretre N, Masullo C, Zerr I, de Silva R, Wientjens DP, et al: Case-control study of risk factors of Creutzfeldt-Jakob disease in Europe during 1993-95. European Union (EU) Collaborative Study Group of Creutzfeldt-Jakob disease (CJD). Lancet 1998;351:1081-1085.

28 Zerr I, Brandel JP, Masullo C, Wientjens DP, de Silva R, Zeidler M, et al: European surveillance on Creutzfeldt-Jakob disease: a casecontrol study for medical risk factors. J Clin Epidemiol 2000;53:747-754.

29 Laske C, Gefeller O, Pfahlberg A, Zerr I, Schroter A, Poser S: The effect of stress on the onset and progression of Creutzfeldt-Jakob disease: results of a German pilot case-control study. Eur J Epidemiol 1999;15:631-635.

30 Nakamura Y, Oki I, Tanihara S, Ojima T, Yanagawa H, Kitamoto T, et al: A case-control study of Creutzfeldt-Jakob disease in Japan: transplantation of cadaveric dura mater was a risk factor. J Epidemiol 2000;10:399_ 402.

-31 Collins S, Law MG, Fletcher A, Boyd A, Kaldor J, Masters CL: Surgical treatment and risk of sporadic Creutzfeldt-Jakob disease: a casecontrol study. Lancet 1999;353:693-697.

32 Ward HJ, Everington D, Croes EA, Alperovitch A, Delasnerie-Laupretre N, Zerr I, et al: Sporadic Creutzfeldt-Jakob disease and surgery: a case-control study using community controls. Neurology 2002;59:543-548.

33 S-Juan P, Ward HJ, de Silva R, Knight RS, Will RG: Ophthalmic surgery and Creutzfeldt-Jakob disease. Br J Ophthalmol 2004;88:446-449.

-34 Ward HJ, Everington D, Cousens SN, SmithBathgate B, Gillies M, Murray K, et al: Risk factors for sporadic Creutzfeldt-Jakob disease. Ann Neurol 2008;63:347-354.

35 Hamaguchi T, Noguchi-Shinohara M, Nozaki I, Nakamura Y, Sato T, Kitamoto T, et al: Medical procedures and risk for sporadic Creutzfeldt-Jakob disease, Japan, 19992008. Emerg Infect Dis 2009;15:265-271.

-36 Ruegger J, Stoeck K, Amsler L, Blaettler T, Zwahlen M, Aguzzi A, et al: A case-control study of sporadic Creutzfeldt-Jakob disease in Switzerland: analysis of potential risk factors with regard to an increased CJD incidence in the years 2001-2004. BMC Public Health 2009;9:18.

-37 Dorsey K, Zou S, Schonberger LB, Sullivan M, Kessler D, Notari E, et al: Lack of evidence of transfusion transmission of CreutzfeldtJakob disease in a US surveillance study. Transfusion 2009;49:977-984.

38 Zaman SM, Hill FG, Palmer B, Millar CM, Bone A, Molesworth AM, et al: The risk of variant Creutzfeldt-Jakob disease among UK patients with bleeding disorders, known to have received potentially contaminated plasma products. Haemophilia 2011;17:931-937.
39 Rothman KJ, Greenland S, Lash TL: Casecontrol studies; in Modern Epidemiology, ed 3. Philadelphia, Lippincott Williams \& Wilkins, 2008, pp 111-127.

40 Wacholder S: The case-control study as data missing by design: estimating risk differences. Epidemiology 1996;7:144-150.

41 Weightman A, Ellis S, Cullum A, Sander L, Turley R: Grading evidence and recommendations for public health interventions: developing and piloting a framework. Health Development Agency 2005, March 31. http:// www.nice.org.uk/download.aspx?o $=503421$ (cited 2010 May 11).

-42 Guyatt GH, Oxman AD, Kunz R, Falck-Ytter Y, Vist GE, Liberati A, et al: Going from evidence to recommendations. BMJ 2008;336: 1049-1051.

43 Ladogana A, Puopolo M, Croes EA, Budka H, Jarius C, Collins S, et al: Mortality from Creutzfeldt-Jakob disease and related disorders in Europe, Australia, and Canada. Neurology 2005;64:1586-1591.

44 Josephs KA, Ahlskog JE, Parisi JE, Boeve BF, Crum BA, Giannini C, et al: Rapidly progressive neurodegenerative dementias. Arch Neurol 2009;66:201-207.

45 Schmidt C, Wolff M, Weitz M, Bartlau T, Korth C, Zerr I: Rapidly progressive Alzheimer disease. Arch Neurol 2011;68:11241130 .

46 Glover JA: The incidence of tonsillectomy in school children. 1938. Int J Epidemiol 2008; 37:9-19.

47 Klemetti A, Saxen L: Prospective versus retrospective approach in the search for environmental causes of malformations. Am J Public Health Nations Health 1967;57:20712075

48 Gravenor MB, Stallard N, Curnow R, McLean AR: Repeated challenge with prion disease: the risk of infection and impact on incubation period. Proc Natl Acad Sci USA 2003;100:10960-10965.

49 Boelle PY, Cesbron JY, Valleron AJ: Epidemiological evidence of higher susceptibility to vCJD in the young. BMC Infect Dis 2004; $4: 26$.

50 Llewelyn CA, Hewitt PE, Knight RS, Amar K, Cousens SN, Mackenzie J, et al: Possible transmission of variant Creutzfeldt-Jakob disease by blood transfusion. Lancet 2004; 363:417-421.

-51 Beeri MS, Rapp M, Silverman JM, Schmeidler J, Grossman HT, Fallon JT, et al: Coronary artery disease is associated with Alzheimer disease neuropathology in APOE4 carriers. Neurology 2006;66:1399-1404.

52 Lee TA, Wolozin B, Weiss KB, Bednar MM: Assessment of the emergence of Alzheimer's disease following coronary artery bypass graft surgery or percutaneous transluminal coronary angioplasty. J Alzheimers Dis 2005;7:319-324. 


\section{Erratum}

In the article by de Pedro Cuesta et al., entitled 'Sensitivity to biases of case-control studies on medical procedures, particularly surgery and blood transfusion, and risk of CreutzfeldtJakob disease' [Neuroepidemiology 2012;39:1-18, DOI: 10.1159/000339318], the authors noticed to their dismay that line 4 of the Introduction contains an error which could very easily mislead the reader.

As published, the phrase in question reads 'CJD exists in three forms: sporadic (sCJD), which is acquired, either variant (vCJD) or iatrogenic (iCJD) CJD, and cases caused by mutations in the gene-encoding $\operatorname{PrP}^{\mathrm{C}}$, here denoted for purposes of simplicity as genetic CJD (gCJD) [1]'. The words 'which is' are clearly erroneous and should be deleted. The sentence correctly reads 'CJD exists in three forms: sporadic (sCJD), acquired, either variant (vCJD) or iatrogenic (iCJD) CJD, and cases caused by mutations in the gene-encoding $\operatorname{PrP}^{\mathrm{C}}$, here denoted for purposes of simplicity as genetic CJD (gCJD) [1]'. 